Pacific Journal of Mathematics

MATRICES WITH PRESCRIBED CHARACTERISTIC
POLYNOMIAL AND A PRESCRIBED SUBMATRIX. 


\title{
MATRICES WITH PRESCRIBED CHARACTERISTIC POLYNOMIAL AND A PRESCRIBED SUBMATRIX
}

\author{
Graciano N. DE Oliveira
}

Let $A$ be an arbitrary (complex) $n \times n$ matrix and let $f(\lambda)$ be a polynomial (with complex coefficients) of degree $n+1$ with leading coefficient $(-1)^{n+1}$. In this paper we solve the problem: under what conditions does there exist an $(n+1) \times(n+1)$ (complex) matrix $B$ of which $A$ is the submatrix standing in the top left-hand corner and such that $f(\lambda)$ is its characteristic polynomial?

In [1] Farahat and Ledermann proved that if $A$ is a nonderogatory matrix over a field $\Phi$ and $f(\lambda)$ is a monic polynomial over $\Phi$, then there exists an $(n+1) \times(n+1)$ matrix $B$ over $\Phi$ with $A$ standing in its top left-hand corner and such that $f(\lambda)=\operatorname{det}\left(\lambda E_{n+1}-B\right)$. Now, our main results are:

Theorem 1. Let $A$ be an $n \times n$ complex matrix whose distinct characteristic roots are $w_{\alpha}(\alpha=1, \cdots, t)$. Let us suppose that in the Jordan normal form of $A, w_{\alpha}$ appears in $r_{\alpha}$ distinct diagonal blocks of orders $v_{1}^{(\alpha)}, \cdots, v_{r_{\alpha}}^{(\alpha)}$ respectively. We assume that

$$
v_{1}^{(\alpha)} \leqq \cdots \leqq v_{r_{\alpha}}^{(\alpha)}
$$

Let $\theta_{\alpha}=\sum_{j=1}^{r_{\alpha}-1} v_{j}^{(\alpha)}$. There exists an $(n+1) \times(n+1)$ complex matrix $B$ having $A$ in the top left-hand corner and with $f(\lambda)$ as characteristic polynomial (i.e., $f(\lambda)=\operatorname{det}\left(B-\lambda E_{n+1}\right)$ ) if and only if $f(\lambda)$ is $d i$ visible by $\prod_{\alpha=1}^{t}\left(w_{\alpha}-\lambda\right)^{\theta}$.

Theorem 2. Let $A$ be a real $n \times n$ symmetric matrix whose distinct characteristic roots are $w_{\alpha}(\alpha=1, \cdots, t)$. Let $r_{\alpha}$ be the multiplicity of $w_{\alpha}$. There exists a real $(n+1) \times(n+1)$ symmetric matrix $B$ having $A$ in the top left-hand corner and with $f(\lambda)$ (now with real coefficients) as characteristic polynomial if and only if

$$
f(\lambda) \text { is divisible by } \prod_{\alpha=1}^{t}\left(w_{\alpha}-\lambda\right)^{r_{\alpha}-1}
$$

and

(b)

$$
\left[\frac{f(\lambda)}{\left(w_{\beta}-\lambda\right)^{r-1}}\right]_{\lambda=\lambda_{\beta}} \cdot \quad \prod_{\substack{\alpha=1 \\ \alpha \neq \beta}}^{t}\left(w_{\alpha}-w_{\beta}\right)^{r_{\alpha}}(\beta=1, \cdots, t)
$$

is real and nonpositive.

REMARK. There is no difficulty in seeing that the conditions (a) 
and (b) imposed on $f(\lambda)$ are equivalent to the following: $f(\lambda)$ has only real roots wich are interlaced by the $n$ characteristic roots of $A$.

2. We start with the following

Lemma. Let $A$ be any $n \times n$ complex matrix with normal Jordan form $J$. In order that the matrix $B$ referred in Theorem 1 exists, it is necessary and sufficient that there should exist a column $X_{1}$ (with $n$ elements), a row $Y_{1}$ (with $n$ elements) and a number $q_{1}$ such that

$$
\left[\begin{array}{ll}
J & X_{1} \\
Y_{1} & q_{1}
\end{array}\right]
$$

has $f(\lambda)$ as characteristic polynomial.

Proof. Let $T$ be an $n \times n$ nonsingular matrix such that $T A T^{-1}=$ $J$. Suppose $B$ exists and is given by

$$
B=\left[\begin{array}{cc}
A & X \\
Y & q
\end{array}\right] .
$$

Let

$$
S=\left[\begin{array}{ll}
T & 0 \\
0 & 1
\end{array}\right] .
$$

We have

$$
S B S^{-1}=\left[\begin{array}{lr}
J & T X \\
Y T^{-1} & q
\end{array}\right] .
$$

and so we can take $Y_{1}=Y T^{-1}, X_{1}=T X$ and $q_{1}=q$.

The converse is easily proved in a similar way.

Our next step is to deduce the characteristic polynomial of the matrix:

$$
C_{i}=\left[\begin{array}{ccccc}
J_{i} & 0 & \cdots & 0 & X_{i} \\
0 & J_{i+1} & \cdots & 0 & X_{i+1} \\
\cdot & \cdot & \cdots & \cdot & \cdot \\
0 & 0 & \cdots & J_{m} & X_{m} \\
Y_{i} & Y_{i+1} & \cdots & Y_{m} & q
\end{array}\right]
$$

where, with obvious notation, 


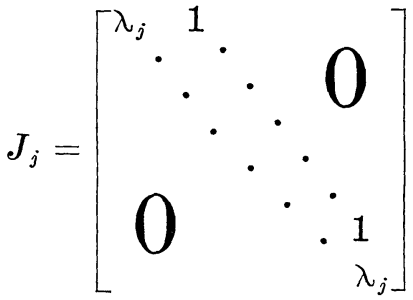

$$
\begin{aligned}
& X_{j}=\left[\begin{array}{c}
x_{1}^{j} \\
\vdots \\
\vdots \\
x_{s_{j}}^{j}
\end{array}\right], \quad Y_{j}=\left[y_{1}^{j}, \cdots, y_{s_{j}}^{j}\right] \quad(j=i, \cdots, m)
\end{aligned}
$$

and $q$ is a complex number.

We expand $\operatorname{det}\left(C_{i}-\lambda E_{i}\right)$ (where $E_{i}$ is the identity matrix of the same order as $C_{i}$ ) by Laplace Theorem in terms of its first $s_{i}$ rows. In order to do this let us find all the nonzero minors contained in these rows. They are: $J_{i} \lambda E^{(i)}\left(E^{(i)}\right.$ denotes the identity matrix of the same order as $J_{i}$ ) and the $s_{i}$ minors formed with $s_{i}-1$ columns of $J_{i}-\lambda E^{(i)}$ and the column $X_{i}$. These $s_{i}$ minors are given by

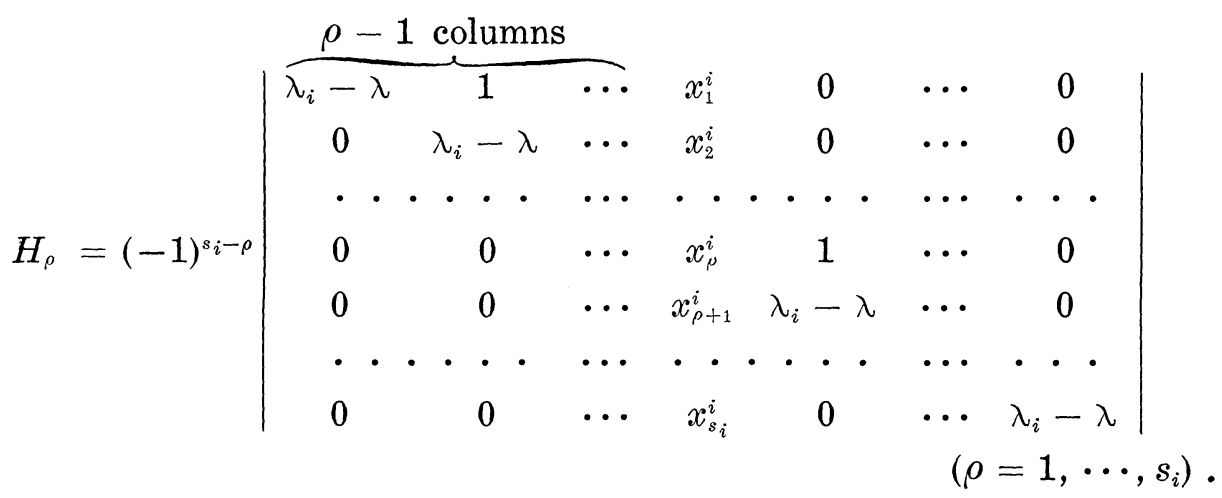

We have

$$
H_{\rho}=(-1)^{s_{i-\rho}}\left(\lambda_{i}-\lambda\right)^{\rho-1} P_{\rho}
$$

with

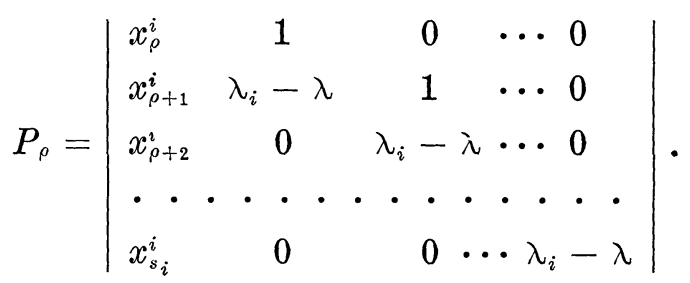

Expanding $P_{\rho}$ in terms of the first row we get 


$$
P_{\rho}=x_{\rho}^{i}\left(\lambda_{i}-\lambda\right)^{s_{i}-\rho}-P_{\rho+1}
$$

and by induction it can be easily seen that

$$
P_{\rho}=\sum_{\tau=0}^{s_{i}-o}(-1)^{\tau} x_{\rho+\tau}^{i}\left(\lambda_{i}-\lambda\right)^{s_{\imath}-\rho-\tau} ;
$$

so we can write

$$
H_{\rho}=\sum_{\tau=0}^{s_{i}-\rho}(-1)^{\tau+s_{i}-\rho} x_{\rho+\tau}^{i}\left(\lambda_{i}-\lambda\right)^{s_{i}-\tau-1} .
$$

Let us now calculate the complementary minor $\widetilde{H}_{\rho}$ of $H_{\rho}$ in $C_{i}-$ $\lambda E_{i}$. There is no difficulty in seeing that

$$
\widetilde{H}_{\rho}=\left|\begin{array}{cccccc}
\overbrace{0}^{\text {column }} J_{i+1}-\lambda E^{(i+1)} & 0 & \cdots & 0 & 0 \\
0 & 0 & J_{i+2}-\lambda E^{(i+2)} & \cdots & 0 & 0 \\
0 & 0 & 0 & \cdots & 0 & 0 \\
\cdot & \cdot & \cdot & \cdots & \cdot & \cdot \\
0 & 0 & 0 & \cdots & 0 & J_{m}-\lambda E^{(m)} \\
y_{\rho}^{i} & Y_{i+1} & Y_{i+2} & \cdots & Y_{m-1} & Y_{m}
\end{array}\right|
$$

We have

$$
\widetilde{H}_{\rho}=(-1)^{\sigma} y_{\rho}^{i} \prod_{j=i+1}^{m}\left(\lambda_{j}-\lambda\right)^{s_{j}}
$$

with

$$
\sigma=\sum_{k=i+1}^{m} s_{k} .
$$

Bearing in mind that $H_{\rho}$ was formed from the rows $1, \cdots, s_{i}$ and columns $1, \cdots, \rho-1, \rho+1, \cdots, s_{i}, \sum_{k=i}^{m} s_{k}+1$, we have

$$
\begin{aligned}
\operatorname{det}\left(C_{i}-\lambda E_{i}\right)= & \sum_{\rho=1}^{s_{i}} \sum_{\tau=0}^{s_{i}-\rho}(-1)^{\tau+1} y_{\rho}^{i} x_{\rho+\tau}^{i}\left(\lambda_{i}-\lambda\right)^{s_{i-\tau-1}} \prod_{j=i+1}^{m}\left(\lambda_{j}-\lambda\right)^{s j} \\
& +\operatorname{det}\left(J_{i}-\lambda E^{i j}\right) \operatorname{det}\left[\operatorname{comp}\left(J_{i}-\lambda E^{(i)}\right)\right],
\end{aligned}
$$

where the symbol comp $\left(J_{i}-\lambda E^{(i)}\right)$ means the complementary minor of $J_{i}-\lambda E^{(i)}$ in the matrix $C_{i}-\lambda E_{i}$. Interchanging the order of the first two sums, noting that $\operatorname{det}\left(J_{i}-\lambda E^{i i}\right)=\left(\lambda_{i}-\lambda\right)^{s_{i}}$ and that comp $\left(J_{i}-\lambda E^{i i}\right)=\operatorname{det}\left(C_{i+1}-\lambda E_{i+1}\right)$ we get

$$
\begin{aligned}
\operatorname{det}\left(C_{i}-\lambda E_{i}\right)= & \sum_{\tau=0}^{s_{i}-1} \sum_{\rho=1}^{s_{i}-\tau}(-1)^{\tau+1} y_{\rho}^{i} x_{\rho+\tau}^{i}\left(\lambda_{i}-\lambda\right)^{s_{i-\tau-1}} \prod_{j=i+1}^{m}\left(\lambda_{j}-\lambda\right)^{s_{j}} \\
& +\left(\lambda_{i}-\lambda\right)^{s_{i}} \operatorname{det}\left(C_{i+1}-\lambda E_{i+1}\right) .
\end{aligned}
$$


Putting here successively $i=1,2, \cdots, m$ and writing for the sake of simplicity

$$
b_{k \mu}=\sum_{\rho=1}^{\mu+1}(-1)^{s_{k}-\mu} y_{\rho}^{k} x_{\rho+s_{k}-1-\mu}^{k} \quad\left(\mu=0, \cdots, s_{k}-1\right),
$$

we get after some manipulation

$$
\begin{aligned}
\operatorname{det}\left(C_{1}-\lambda E_{1}\right)= & \sum_{k=1}^{m}\left\{\left[\sum_{j=0}^{s_{k}-1} b_{k \mu}\left(\lambda_{k}-\lambda\right)^{\mu}\right]\left[\prod_{\substack{j=1 \\
j \neq k}}^{m}\left(\lambda_{j}-\lambda\right)^{s_{j}}\right]\right\} \\
& +(q-\lambda) \prod_{j=1}^{m}\left(\lambda_{j}-\lambda\right)^{s_{j}} .
\end{aligned}
$$

We are now ready for the proof of Theorem 1. Because of the lemma it is sufficient to prove the theorem assuming that $A$ is in the Jordan normal form $J=\operatorname{diag}\left(J_{1}, \cdots, J_{m}\right)$ with $J_{j}(j=1, \cdots, m)$ given by (2.2). So what we have to do is to find out under what conditions it is possible to find columns $X_{1}, \cdots, X_{m}$, rows $Y_{1}, \cdots, Y_{m}$ and a number $q$ such that the characteristic polynomial (2.4) of the matrix $C_{1}$ be $f(\lambda)$.

As in the Jordan normal form the order in which the diagonal blocks occur is arbitrary, we can suppose without loss of generality that

$$
\begin{aligned}
\lambda_{1} & =\cdots=\lambda_{u_{1}} \quad\left(=w_{1}\right) \\
\cdot & \cdots \cdot \cdot \cdot \\
\lambda_{u_{\alpha-1}+1} & \cdots=\lambda_{u_{\alpha}} \quad\left(=w_{\alpha}\right) \\
\cdot & \cdots \cdot \cdot \cdot \cdot \\
\lambda_{u_{t-1}+1} & \cdots=\lambda_{u_{t}} \quad\left(=w_{t}\right) \\
& \quad\left(u_{\alpha}=\sum_{\beta=1}^{\alpha} r_{\beta} ; r_{\beta}\right. \text { defined in Theorem 1) }
\end{aligned}
$$

with $w_{\alpha} \neq w_{\beta}$ if $\alpha \neq \beta$. With this notation, in $J$ the characteristic root $w_{\alpha}$ appears in the diagonal blocks $J_{u_{\alpha-1}+1} \cdots, J_{u_{\alpha}}$ which are of orders $s_{u_{\alpha-1}+1} \cdots, s_{u_{\alpha}}$ respectively. We will assume that

$$
s_{u_{\alpha-1}+1} \leqq \cdots \leqq s_{u}
$$

for every $\alpha$.

Let

$$
\theta_{\alpha}=\sum_{\mu=u_{\alpha-1}+1}^{u_{\alpha}-1} s_{\mu^{\prime}}
$$

From (2.4) we have

$$
\operatorname{det}\left(C_{1}-\lambda E_{1}\right)=\left(w_{\alpha}-\lambda\right)^{\theta} \varphi_{\alpha}(\lambda)
$$

where $\varphi_{\alpha}(\lambda)$ is a polynomial in $\lambda$ which is not necessarily divisible by $w_{\alpha}-\lambda$. As $\alpha \neq \beta$ implies $w_{\alpha} \neq w_{\beta}$ we will have 


$$
\operatorname{det}\left(C_{1}-\lambda E_{1}\right)=\prod_{\alpha=1}^{t}\left(w_{\alpha}-\lambda\right)^{\theta_{\alpha}} \psi(\lambda)
$$

where $\psi(\lambda)$ is a polynomial in $\lambda$ not necessarily divisible by any factor of $h(\lambda)=\prod_{\alpha=1}^{t}\left(w_{\alpha}-\lambda\right)^{\theta}$. Therefore, if $f(\lambda)$ is not divisible by $h(\lambda)$ it is impossible to find $X_{i}, Y_{i}(i=1, \cdots, m)$ and $q$ such that $f(\lambda)=$ $\operatorname{det}\left(C_{1}-\lambda E_{1}\right)$. Let us now suppose that $f(\lambda)=h(\lambda) f_{1}(\lambda)$. All we have to prove is that it is possible to find $X_{i}, Y_{i}(i=1, \cdots, m)$ and $q$ such that $\psi(\lambda)=f_{1}(\lambda)$.

Setting

$$
S_{k}(\lambda)=\sum_{\mu=0}^{s_{k}-1} b_{k \mu}\left(\lambda_{k}-\lambda\right)^{\mu}
$$

and

$$
\xi_{\alpha}=\sum_{\mu=u_{\alpha-1}+1}^{u_{\alpha}} s_{\mu},
$$

(2.4) gives

$$
\begin{aligned}
\operatorname{det}\left(C_{1}-\lambda E_{1}\right)= & \sum_{\beta=0}^{t-1} \sum_{k=u_{\beta}+1}^{u_{\beta+1}} S_{k}(\lambda) \frac{\prod_{\alpha=1}^{t}\left(w_{\alpha}-\lambda\right)^{\xi_{\alpha}}}{\left(w_{\beta+1}-\lambda\right)^{s_{k}}} \\
& +(q-\lambda) \prod_{\alpha=1}^{t}\left(w_{\alpha}-\lambda\right)^{\xi_{\alpha}} \quad\left(u_{0}=0\right) .
\end{aligned}
$$

Let us choose $b_{k^{\mu}}=0$ for every $k \neq u_{\beta+1}(\beta=0, \cdots, t-1 ; \mu=0, \cdots$, $\left.s_{k}-1\right)$. With this choice (2.7) gives

$$
\begin{aligned}
\operatorname{det}\left(C_{1}-\lambda E_{1}\right)= & \prod_{\gamma=1}^{t}\left(w_{\gamma}-\lambda\right)^{\theta_{\gamma}}\left[\sum_{\beta=0}^{t-1} S_{u_{\beta+1}}(\lambda) \prod_{\substack{\alpha=1 \\
\alpha \neq \beta+1}}^{t}\left(w_{\alpha}-\lambda\right)^{s_{u_{\alpha}}}\right. \\
& \left.+(q-\lambda) \prod_{\alpha=1}^{t}\left(w_{\alpha}-\lambda\right)^{\xi_{\alpha}-\theta_{\alpha}}\right]
\end{aligned}
$$

and so by (2.5)

$$
\psi(\lambda)=\sum_{\beta=0}^{t-1} S_{u_{\beta+1}}(\lambda) \prod_{\substack{\alpha=1 \\ \alpha \neq \beta+1}}^{t}\left(w_{\alpha}-\lambda\right)^{s} u_{\alpha}+(q-\lambda) \prod_{\alpha=1}^{t}\left(w_{\alpha}-\lambda\right)^{s} u_{\alpha} .
$$

By (2.6) $S_{u_{\beta+1}}(\lambda)$ is a polynomial in $\left(w_{\beta+1}-\lambda\right)$ of degree $s_{u_{\beta+1}}-1$. For the sake of simplicity we now change the notation (in an obvious way) writing

$$
\psi(\lambda)=\sum_{\beta=0}^{i-1} R_{\beta}(\lambda) \prod_{\substack{\alpha=1 \\ \alpha \neq \beta+1}}^{t}\left(w_{\alpha}-\lambda\right)^{t}+(q-\lambda) \prod_{\alpha=1}^{t}\left(w_{\alpha}-\lambda\right)^{t} \alpha
$$

Let

$$
R_{\beta}(\lambda)=\sum_{\mu=0}^{t_{\beta+1}-\tau} \delta_{\beta \mu}\left(w_{\beta+1}-\lambda\right)^{\mu}
$$

We can write 


$$
\frac{\psi(\lambda)}{\prod_{\alpha=1}^{t}\left(w_{\alpha}-\lambda\right)^{t_{\alpha}}}=\sum_{\beta=0}^{t-1} \sum_{\mu=0}^{t_{\beta+1}-1} \frac{\delta_{\beta \mu}}{\left(w_{\beta+1}-\lambda\right)^{t_{\beta+1}-\mu}}+q-\lambda .
$$

Let us resolve $f_{1}(\lambda) / \prod_{\alpha=1}^{t}\left(w_{\alpha}-\lambda\right)^{t} \alpha$ into partial fractions. We will get

$$
\frac{f_{1}(\lambda)}{\prod_{\alpha=1}^{t}\left(w_{\alpha}-\lambda\right)^{t_{\alpha}}}=\sum_{\beta=0}^{t-1} \sum_{\mu=0}^{t_{\beta+1}} \frac{A_{\beta \mu}}{\left(w_{\beta+1}-\lambda\right)^{t_{\beta+1}-\mu}}+Q-\lambda .
$$

If now in (2.8) we take $\delta_{\beta \mu}=A_{\beta \mu}$ and $q=Q$ we will have $\psi(\lambda)=$ $f_{1}(\lambda)$ as required. So we have given a process to choose all the $b_{k \mu}$ appearing in (2.6). To conclude the proof we show that it is always possible to find valuse $x_{\sigma}^{i}, y_{\sigma}^{i}$ satisfying (2.3), no matter what values we have given to the $b_{k \mu}$. In fact, let us give to the $x_{o}^{i}$ arbitrary nonzero values ( $x_{\sigma}^{i}=1$, for example). Then, for each $k,(2.3)$ becomes a system of linear equations in the $y_{\rho}^{k}$ with a triangular matrix whose principal elements are different from zero. This means that the system is compatible. The proof of Theorem 1 is now complete.

CoRollary. If $A$ is a complex nonderogatory matrix, then the matrix $B$ of Theorem 1 always exists.

Proof. If $A$ is nonderogatory in its Jordan normal form there are no two diagonal blocks corresponding to the same characteristic root. So in Theorem 1 we have $r_{\alpha}=1$ and so $\theta_{\alpha}=0$. This means that $B$ exists.

Proof of Theorem 2. If $A$ is real and symmetric, the matrix $T$ such that $T A T^{-1}=J$ can be chosen orthogonal and $J$ will be a diagonal matrix. So using Theorem 1 we have $v_{1}^{\langle\alpha\rangle}=\cdots=v_{r_{\alpha}}^{(\alpha)}=1$ and $\theta_{\alpha}=r_{\alpha}-1$. It follows that (a) is necessary and sufficient for the existence of a matrix $B$ (not necessarily real and symmetric) of type $(n+1) \times(n+1)$ having $A$ in the top left-hand corner and with $f(\lambda)$ as characteristic polynomial. Let us now find out the conditions for $B$ to be real and symmetric. Choosing $T$ orthogonal for $B$ to fulfill this condition it is necessary and sufficient that there exist real $X_{j}, Y_{j}, q(j=1, \cdots, m)$ with $X_{j}=Y_{j}$. Let us write $x_{\rho}^{i}=$ $y_{\rho}^{i}$. We have now $\xi_{\alpha}=r_{\alpha}, \theta_{\alpha}=\xi_{\alpha}-1$ and $S_{k}(\lambda)=b_{k 0}$. Let

$$
c_{\beta 0}=\sum_{k=u_{\beta}+1}^{u_{\beta+1}} b_{k 0} .
$$

The formula (2.7) gives 
$\operatorname{det}\left(C_{1}-\lambda E_{1}\right)=\prod_{\gamma=1}^{t}\left(w_{\gamma}-\lambda\right)^{r_{\gamma}-1}\left[\sum_{\beta=0}^{t-1} c_{\beta 0} \prod_{\substack{\alpha=1 \\ \alpha \neq \beta+1}}^{t}\left(w_{\alpha}-\lambda\right)+(q-\lambda) \prod_{\alpha=1}^{t}\left(w_{\alpha}-\lambda\right)\right]$ and so

$$
\psi(\lambda)=\sum_{\beta=0}^{t-1} c_{\beta 0} \prod_{\substack{\alpha=1 \\ \alpha \neq \beta+1}}^{t}\left(w_{\alpha}-\lambda\right)+(q-\lambda) \prod_{\alpha=1}^{t}\left(w_{\alpha}-\lambda\right)
$$

We are assuming that $f(\lambda)$ is divisible by

$$
h(\lambda)=\prod_{\alpha=1}^{t}\left(w_{\alpha}-\lambda\right)^{r_{\alpha}-1}
$$

Let $f(\lambda) / h(\lambda)=f_{1}(\lambda)$. Resolving $f_{1}(\lambda) / \prod_{\alpha=1}^{t}\left(w_{\alpha}-\lambda\right)$ into partial fractions we get

$$
\frac{f_{1}(\lambda)}{\prod_{\alpha=1}^{t}\left(w_{\alpha}-\lambda\right)}=\sum_{\beta=0}^{t-1} \frac{B_{\beta}}{w_{\beta+1}-\lambda}+Q_{1}-\lambda
$$

with

$$
B_{\beta}=\frac{f_{1}\left(w_{\beta+1}\right)}{\prod_{\substack{\alpha=1 \\ \alpha \neq \beta+1}}^{t}\left(w_{\alpha}-w_{\beta+1}\right)}
$$

From (2.10) we have

$$
\frac{\psi(\lambda)}{\prod_{\alpha=1}^{t}\left(w_{\alpha}-\lambda\right)}=\sum_{\beta=0}^{t-1} \frac{c_{\beta 0}}{w_{\beta+1}-\lambda}+q-\lambda
$$

So we must take

$$
c_{\beta 0}=\frac{f_{1}\left(w_{\beta+1}\right)}{\prod_{\substack{\alpha=1 \\ \alpha \neq \beta+1}}^{t}\left(w_{\alpha}-w_{\beta+1}\right)}, q=Q_{1}
$$

The equations (2.3) now take the form

$$
b_{k 0}=-\left[x_{1}^{k}\right]^{2}
$$

or, by $(2.9)$

$$
c_{\beta 0}=-\sum_{k=u_{\beta}+1}^{u_{\beta+1}}\left[x_{1}^{k}\right]^{2} .
$$

So $B$ can be real and symmetric if and only if $c_{\beta 0} \leqq 0$ and $Q_{1}$ is real. The condition $c_{\beta 0} \leqq 0$ is equivalent to (b). Bearing in mind that $\sum_{\alpha=1}^{t} w_{\alpha}$ is real we can see easily that $Q_{1}$ is always real. With this the proof is complete. 
In a similar way we could prove a theorem analogous to Theorem 2 but with 'real symmetric' substituted by 'hermitian'.

Note. After I had written this paper I noticed that Theorem 2 is not new. It is essentially equivalent to Theorem 1 in Fan and Pall, Imbedding Conditions for Hermitian and Normal Matrices, Canad. J. Math. 9 (1957), 298-304. However, the proof I have given here is a bit different from the proof of Fan and Pall. For further details see my forthcoming paper Matrices with prescribed characteristic polynomial and a prescribed submatrix-II (submitted to Pacific J. Math.).

I wish to thank the referee for his comments.

\section{REFERENCE}

1. Farahat and Ledermann, Matrices with prescribed characteristic polynomial, Proc. Edinburgh Math. Soc. (2) 11 (1959), 143-146.

Received March 5, 1968.

UNIVERSIDADE DE COIMBRA

CoImbra, Portugal 



\section{PACIFIC JOURNAL OF MATHEMATICS}

\section{EDITORS}

H. ROYDEN

Stanford University

Stanford, California

\author{
R. R. Phelps \\ University of Washington \\ Seattle, Washington 98105
}

J. DugundJI

Department of Mathematics

University of Southern California

Los Angeles, California 90007

RICHARD ARENS

University of California

Los Angeles, California 90024

\section{ASSOCIATE EDITORS}

E. F. BECKenbaCH

B. H. NEUMANN

F. WOLF

K. YoSHIDA

\section{SUPPORTING INSTITUTIONS}

\author{
UNIVERSITY OF BRITISH COLUMBIA \\ CALIFORNIA INSTITUTE OF TECHNOLOGY \\ UNIVERSITY OF CALIFORNIA \\ MONTANA STATE UNIVERSITY \\ UNIVERSITY OF NEVADA \\ NEW MEXICO STATE UNIVERSITY \\ OREGON STATE UNIVERSITY \\ UNIVERSITY OF OREGON \\ OSAKA UNIVERSITY \\ UNIVERSITY OF SOUTHERN CALIFORNIA
}

\author{
STANFORD UNIVERSITY \\ UNIVERSITY OF TOKYO \\ UNIVERSITY OF UTAH \\ WASHINGTON STATE UNIVERSITY \\ UNIVERSITY OF WASHINGTON \\ $\stackrel{*}{*} \stackrel{*}{*} \stackrel{*}{*}$ AMERICAN MATHEMATICAL SOCIETY \\ CHEVRON RESEARCH CORPORATION \\ TRW SYSTEMS \\ NAVAL WEAPONS CENTER
}

The Supporting Institutions listed above contribute to the cost of publication of this Journal, but they are not owners or publishers and have no responsibility for its content or policies.

Mathematical papers intended for publication in the Pacific Journal of Mathematics should be in typed form or offset-reproduced, double spaced with large margins. Underline Greek letters in red, German in green, and script in blue. The first paragraph or two must be capable of being used separately as a synopsis of the entire paper. It should not contain references to the bibliography. Manuscripts, in duplicate if possible, may be sent to any one of the four editors. Please classify according to the scheme of Math. Rev. 36, 1539-1546. All other communications to the editors should be addressed to the managing editor, Richard Arens, University of California, Los Angeles, California, 90024.

50 reprints are provided free for each article; additional copies may be obtained at cost in multiples of 50 .

The Pacific Journal of Mathematics is published monthly. Effective with Volume 16 the price per volume (3 numbers) is $\$ 8.00$; single issues, $\$ 3.00$. Special price for current issues to individual faculty members of supporting institutions and to individual members of the American Mathematical Society: $\$ 4.00$ per volume; single issues $\$ 1.50$. Back numbers are available.

Subscriptions, orders for back numbers, and changes of address should be sent to Pacific Journal of Mathematics, 103 Highland Boulevard, Berkeley, California, 94708.

PUBLISHED BY PACIFIC JOURNAL OF MATHEMATICS, A NON-PROFIT CORPORATION

Printed at Kokusai Bunken Insatsusha (International Academic Printing Co., Ltd.), 7-17, Fujimi 2-chome, Chiyoda-ku, Tokyo, Japan. 


\section{Pacific Journal of Mathematics \\ Vol. 29, No. $3 \quad$ July, 1969}

Herbert James Alexander, Extending bounded holomorphic functions from certain subvarieties of a polydisc ...................... 485

Edward T. Cline, On an embedding property of generalized Carter

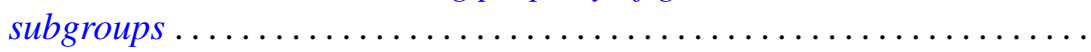

Roger Cuppens, On the decomposition of infinitely divisible characteristic functions with continuous Poisson spectrum. II ...............

William Richard Emerson, Translation kernels on discrete Abelian

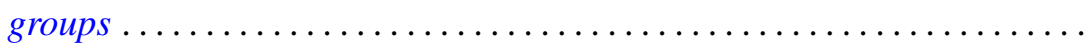

Robert William Gilmer, Jr., Power series rings over a Krull domain ....... 543

Julien O. Hennefeld, The Arens products and an imbedding theorem ...... 551

James Secord Howland, Embedded eigenvalues and virtual poles ........ 565

Bruce Ansgar Jensen, Infinite semigroups whose non-trivial homomorphs are all isomorphic .............................. 583

Michael Joseph Kascic, Jr., Polynomials in linear relations. II .......... 593

J. Gopala Krishna, Maximum term of a power series in one and several

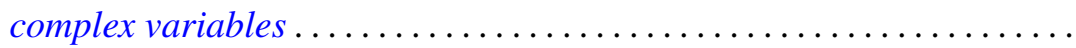

Renu Chakravarti Laskar, Eigenvalues of the adjacency matrix of cubic lattice graphs ...................................

Thomas Anthony Mc Cullough, Rational approximation on certain plane

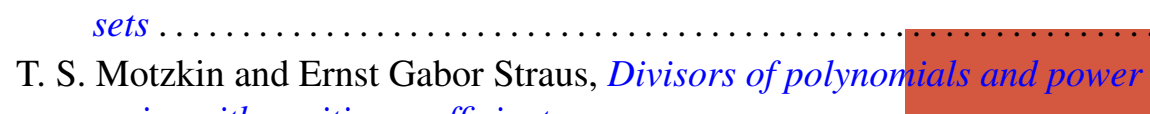
series with positive coefficients .

Graciano de Oliveira, Matrices with prescribed characteristic polynomial and a prescribed submatrix.

Graciano de Oliveira, Matrices with prescribed characteristic polynomial and a prescribed submatrix. II .

Donald Steven Passman, Exceptional 3/2-transitive permutation groups .................................

Grigorios Tsagas, A special deformation of the metric with no negative sectional curvature of a Riemannian space............

Joseph Zaks, Trivially extending decompositions of $E^{n}$ 